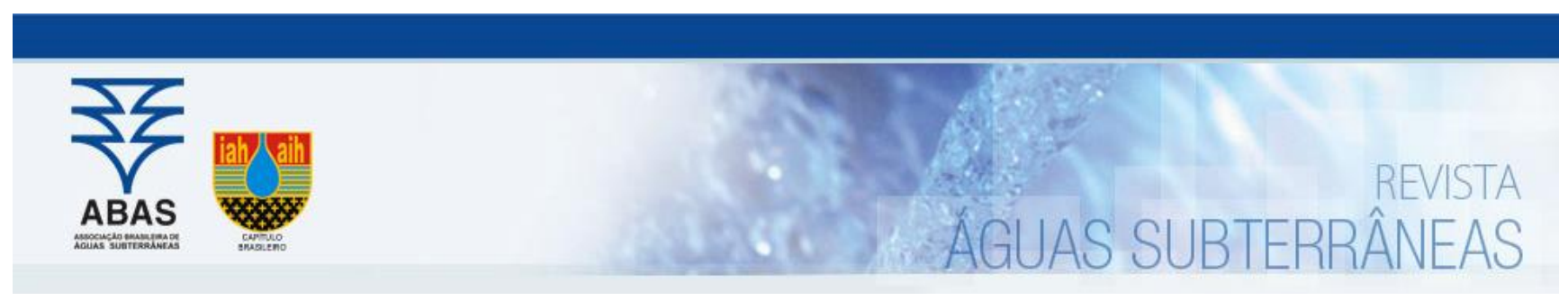

Artigos

\title{
Relação entre precipitação e recarga de águas subterrâneas na Amazônia Central
}

\section{Relationship between precipitation and groundwater recharge in the Central Amazon}

Alderlene Pimentel de Brito1; Javier Tomasella2; Ingo Daniel Wahnfried33; Luiz Antonio Candido1; Maria Terezinha Monteiro1; Sávio José Ferreira Filgueiras ${ }^{1}$

1 Instituto Nacional de Pesquisas da Amazônia

2 Centro Nacional de Monitoramento e Alertas de Desastres Naturais

3 Universidade Federal do Amazonas

alderlenebrito@gmail.com, javier.tomasella@camaden.gov.br, iwahnfried@gmail.com, luiz.antonio.candido@gmail.com, mtmont.10@gmail.com, savio@inpa.gov.br

Palavras-chave:

Variação do nível da água. Pprecipitação e recarga.

Resumo

A água subterrânea na Amazônia é de fundamental importância na manutenção dos serviços ecossistêmicos e para atender demandas humanas. Apesar da existência de grandes corpos de água doce na região, as demandas de abastecimento são atendidas a partir de fontes subterrâneas, majoritariamente freáticas. De forma geral, demandas crescentes do recurso subterrâneo associadas ao crescimento demográfico e padrões de uso do solo podem induzir a alterações nas relações de balanço hídrico e, presumidamente também, as relações entre precipitação-recarga. Somam-se, a esses fatores, efeitos dos eventos climáticos interanuais como o ENOS em suas distintas manifestações: El Niño e La Niña. O objetivo deste estudo é correlacionar as recargas anuais e episódicas nos aquíferos Alter do Chão e Trombetas com a precipitação entre 2011 a 2018, tendo como foco especial o evento de El Niño de 2015-2016. A análise consiste na estimativa das taxas de recarga a partir do método Variação do Nível de Água (VNA) e na comparação das variações do nível da água subterrânea ao déficit total no armazenamento de água para o período. Padrões de comportamento interanuais do nível freático correlacionam-se com a variabilidade observada para a precipitação. A recarga média anual para os aquíferos Alter do Chão e Trombetas foi de 35\% (572 mm) para uma precipitação anual de $1647 \mathrm{~mm}$, e $24 \%$, ou seja, $473 \mathrm{~mm}$ de $1954 \mathrm{~mm}$ precipitados, respectivamente. Os níveis freáticos mínimos ocorreram em janeiro de 2016. Este efeito indica que o ENOS pode gerar diminuições no volume total de água armazenado em aquíferos na Amazônia durante e alguns meses após sua ocorrência.

\section{Keywords:}

Water table fluctuation.

Precipitation, and recharge.

Revisado por pares.

Recebido em: 11/09/2019.

Aprovado em: 12/12/2019.

Abstract

Groundwater in the Amazon is of fundamental importance in maintaining ecosystem services and meeting human demands. Despite the existence of large freshwater bodies in the region, social requests are fulfilled mostly from groundwater sources. In general, increasing groundwater demands associated with demographic growth and land use patterns may have an impact on the overall water availability and presumably in the rainfall-recharge relationships. The effects of interannual weather events such as ENSO, in their different manifestations (El Niño and La Niña), are added to these factors. This study aims to correlate the annual and episodic recharges in Alter do Chão and Trombetas aquifers with precipitation between 2011 and 2018, having as a particular focus the El Niño event of 2015-2016. The analysis consists of estimating the recharge rates obtained from the Water Table Fluctuation method (WTF) and comparing groundwater table variations to the total water storage deficit for the period in this area. Patterns of interannual groundwater behavior correlate with the variability observed for precipitation. The average annual recharge for both aquifers, the Alter do Chão and the Trombetas, were $35 \%(572 \mathrm{~mm})$ of the annual rainfall of $1647 \mathrm{~mm}$ and $24 \%(473 \mathrm{~mm})$ of the yearly rain of $1954 \mathrm{~mm}$ precipitated respectively. The minimum water levels occurred in January of 2016 and are associated with the effects of ENSO's phenomena.

DOI: http:/dx.doi.org/10.14295/ras.v34i1.29616

\section{INTRODUÇÃO}

Aquíferos freáticos são de fundamental importância para as populações amazônicas, embora haja grande disponibilidade hídrica superficial. Tal fato se deve à facilidade de obtenção do recurso, pois o nível médio da água subterrânea para a região é predominantemente raso (FAN; LEE e MACHO, 2013).
A profundidade do lençol freático é variável: varia de zero, quando está na superfície na forma de surgência, a centenas ou até milhares de metros em alguns tipos de aquíferos. Usualmente, a profundidade do lençol freático é pequena perto de corpos permanentes de águas superficiais, como córregos, lagos e pântanos. E sua configuração varia sazonalmente porque a recarga das águas subterrâneas, entendida como a acu- 
mulação de água na superfície superior da zona saturada, está relacionada à variação na quantidade e distribuição temporal de precipitação (WINTER et al., 1999).

Uma sucessão de anos com precipitação superior ou inferior à normal climatológica da região pode gerar longos períodos, de vários anos, com níveis de água subterrânea elevados ou reduzidos em relação a normal piezométrica (VAN CAMP e WALREAVENS, 2013). As implicações do processo recém descrito afetam a sustentação dos serviços ecossistêmicos dependentes das águas subterrâneas. (KLOVE et al., 2014) e a evolução hidrodinâmica dos aquíferos em termos de recarga futura (VAN CAMP e WALREAVENS, 2013). Mudanças nos regimes de precipitação, temperatura e nos padrões de uso do solo são alguns dos principais fatores que controlam as recargas futuras (HOLMAN, 2006). Uma vez associados a pressões antrópicas, como por exemplo a de superexplotação aquífera, tendem a contribuir na diminuição da oferta do recurso.

Nos ecossistemas tropicais, a variabilidade de chuvas afeta todos os componentes de equilíbrio da água (TOMASELLA et al., 2008), formando ciclos plurianuais de deficiência e excesso de chuvas em toda a região amazônica. Produzem-se de tempos em tempos, secas e inundações (MARENGO e ESPINOZA, 2016), que para o caso da região amazônica, são de fundamental importância no funcionamento do clima regional e global (MARENGO, 2004).

Sabe-se que a variabilidade climática de alta frequência (sinótica a sazonal) cria respostas hidrológicas de curto prazo, mas os níveis de água subterrânea e recarga são parcialmente controlados por interações complexas de anomalia meteorológica de baixa frequência (interanual a multidecadal) (TREMBLAY et al., 2011; VENENCIO e GARCIA, 2011; PEREZ-VALDIVIA et al., 2012).

A anomalia meteorológica de baixa frequência controla parcialmente a distribuição da precipitação no espaço e no tempo, a frequência e a severidade das secas, o escoamento dos canais e outros processos hidrológicos superficiais. Entretanto, seus efeitos, no que tange às taxas de recarga e outros processos hidrológicos subterrâneos que afetam a quantidade e a qualidade das águas subterrâneas, ainda são desconhecidos (KUSS e GUDARK, 2014).

Logo, uma melhor compreensão das flutuações de longo prazo na disponibilidade de água subterrânea é essencial para nortear gestores e decisões políticas, particularmente no contexto do uso crescente de água subterrânea para consumo humano e irrigação (WADA et al., 2010) e das incertezas impostas pelas mudanças climáticas (STOLL et al., 2011; GURDAK et al., 2012).

A partir de 1970, conforme Seneviratne et al. (2012) e Gloor et al. (2015), nota-se uma tendência de aumento da temperatura e intensificação da frequência e duração das secas na região amazônica. Modelos climáticos sugerem inclusive que este cenário pode se agravar (MARENGO et al., 2013; GLOOR et al., 2015). Marengo e Espinoza (2016) e Aragão et al. (2018) sugerem que na região Amazônica, estas secas estariam associadas ao aquecimento da temperatura na superfí- cie do mar do Oceano Pacífico Tropical e/ou do Oceano Atlântico Norte Tropical, fenômeno conhecido como El Niño.

No intervalo de tempo transcorrido recente, destacam-se três eventos de secas extremas: 2005, 2010 e 2015-2016 (ARAGÃo et al., 2018). Com relação à seca de 2005 e seus impactos no armazenamento de água subterrânea, Frappart et al. (2019) sugerem que tenha sido induzida por uma anomalia interanual, que resultou em déficit no armazenamento de águas subterrâneas.

A seca de 2015 foi a mais extrema do século XXI, afetando uma área de $1.888 .488 \mathrm{~km}^{2}, 43 \%$ do bioma da Amazônia brasileira em comparação a de 2005 (22\%) e 2010 (25\%). A Amazônia Central e Oriental foram especialmente afetadas (ARAGÃO et al., 2018).

Observa-se que na Amazônia, os impactos das variações interanuais sobre o comportamento dos aquíferos freáticos não é de todo conhecido, principalmente pela baixa densidade da malha de monitoramento e o curto período das observações. Neste contexto, o monitoramento das águas subterrâneas constitui uma ferramenta importante para o entendimento da dinâmica da aquífera e a gestão dos aquíferos (HEALY e COOK, 2002). As estimativas das recargas acabam determinando 0 dimensionamento das reservas exploráveis. Tais resultados podem ser aplicados ao abastecimento público, obras de saneamento entre outros, a depender da demanda econômica (BAUN, MARUSCO e FRITZEN, 2018).

A recarga pode ser classificada em três tipos em função de suas características e distribuição espacial: direta, indireta e localizada. A direta ocorre no local do impacto da precipitação por movimento de infiltração vertical através da zona não-saturada. A recarga indireta ocorre através da percolação das águas superficiais dos leitos dos cursos d'água para o lençol freático. Já a recarga localizada é uma forma intermediária de recarga, resultante da concentração quase horizontal de água superficial na ausência de canais bem definidos (LERNER et al., 1990). Em ambientes urbanos esta complexidade de interrelações relacionadas às recargas é ainda maior, seja por conta da distribuição do escoamento, seja por conta dos vazamentos do sistema de abastecimento (LERNER, 2002).

O objetivo do presente estudo é correlacionar a precipitação e as recargas anuais e episódicas para o período 2010-2018 nos aquíferos Alter do Chão e Trombetas, com foco especial ao evento de El Niño de 2015. A análise baseia-se na avaliação das taxas de recarga anuais e episódicas, obtidas a partir do método de variação do nível da água (VNA), para poços de monitoramento localizados na Amazônia Central e na comparação das variações do nível da água subterrânea dos poços com o déficit total no armazenamento de água para o período.

\section{2. ÁREA DE MONITORAMENTO}

O estudo foi desenvolvido em áreas onde afloram a Formação Alter do Chão (FAC) e o Grupo Trombetas (GT), consideradas detentoras de propriedades aquíferas. FAC é constituída por arenitos médios a grossos avermelhados, arenitos médios a grossos cauliníticos de coloração branco-acinzentada, siltitos 
e argilitos de coloração avermelhada e conglomerados (CUNHA et al., 2007). No contexto hidrogeológico, Aguiar et al. (2002) definiram uma espessura média de $200 \mathrm{~m}$ para a unidade aquífera da FAC na região de Manaus e obtiveram níveis freáticos médios de $25 \mathrm{~m}$ de profundidade, com direção predominante de fluxo de NE para SW. A partir de dados hidrogeoquímicos e datação por métodos isotópicos, Pita et al. (2018) separam a Formação Alter do Chão em dois sistemas aquíferos: (i) 0 aquífero superior (até $130 \mathrm{~m}$ de profundidade) e, (ii) um aquífero inferior (abaixo de $130 \mathrm{~m}$ de profundidade). No aquífero superior a recarga é de origem meteórica, e as águas pouco mineralizadas, enquanto que no sistema aquífero inferior as águas apresentam maior mineralização devido ao maior tempo de residência no aquífero e a um processo de interação água-rocha mais desenvolvido. Essas águas estão armazenadas, geralmente, nas camadas aquíferas mais profundas (mais de $130 \mathrm{~m}$ de profundidade), em condições de confinamento ou semiconfinamento.

O GT ocorre na borda norte da região onde afloram as unidades da Formação Alter do Chão e suas unidades aquíferas estão contidas em estratos arenosos, intercaladas por folhelhos, diamicititos e pelitos (CUNHA et al., 2007). Da base para o topo, o grupo subdivide-se nas formações Autás - Mirim (FAM), Nhamundá (FN), Pitinga (FP) e Manacapuru (FM) (CAPUTO et al., 1984). FAM é a unidade basal deste grupo, representada por intercalação de arenitos, siltitos e folhelhos (ritmitos), não aflorantes na Bacia do Amazonas (CPRM, 2004). FN está constituída por arenitos finos a médios, com subordinada intercalação de folhelho, siltito e diamictito na proximidade do topo da seção (CPRM, 2004). FP é composta de foIhelho/ siltito e diamictito de ambiente glácio-marinho (CUNHA et al., 1994). FM reúne arenitos finos a médios intercalados com siltitos acinzentados e laminados (CUNHA et al., 1994). Com relação às propriedades aquíferas, o Aquífero Trombetas (AT) é tido como poroso, contínuo, de caráter regional e apresenta produtividade baixa a localmente moderada. Há pouca informação do ponto de vista hidrogeológico sobre esse aquífero. Com relação à qualidade, as águas são próprias para consumo humano e apesentam baixo teor em sais dissolvidos com Ph variando entre 4,5 a 5,5, (CPRM, 2014). A Figura 1 apresenta a localização da área de estudo.

Figura 1 - Localização dos poços RIMAS no contexto da Bacia Amazônica com destaque para os aquíferos Alter do Chão e Trombetas. Os poços 1, 2 e 3 localizam-se no município de Manaus (AAC) e os poços 7 e 8 em Presidente Figueiredo (AT), no estado do Amazonas. Os Poços 4, 5 e 5, nos municípios de Santarém, Monte Alegre e Óbidos, na porção do aquífero Alter do Chão no estado do Pará

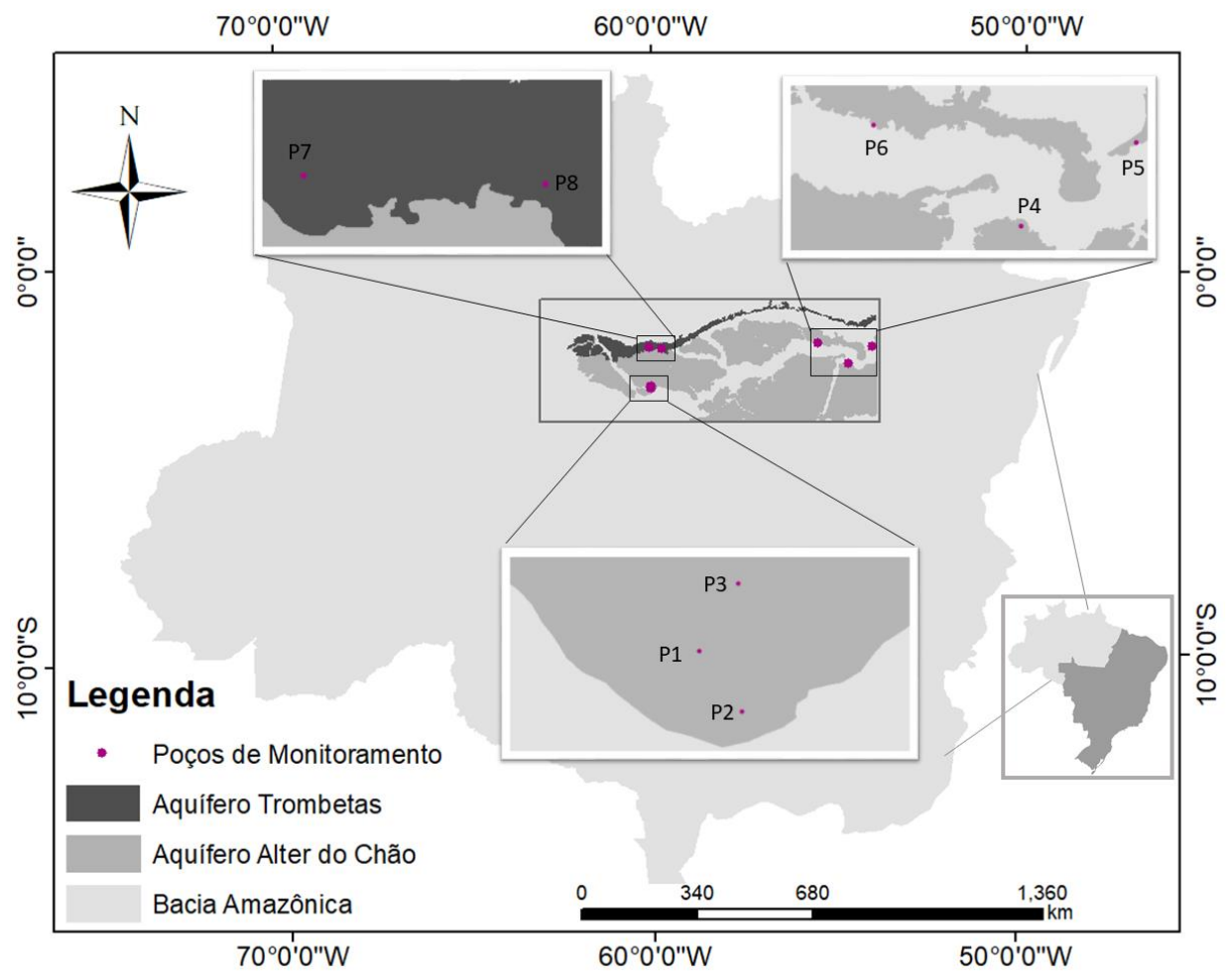

\section{MATERIAIS E MÉTODOS}

\subsection{Dados}

Os dados de precipitação aplicados nesta pesquisa foram disponibilizados pelo INMET (Instituto Nacional de Meteorologia) para os municípios de Manaus - Amazonas (AM), Santarém, Monte Alegre e Óbidos - Pará (PA), no período de 2010 a 2017(http://www.inmet.gov.br/portal/index.php? $r=b d m e p / b d m e p)$. A precipitação de referência para
Manaus foi tomada no município de Presidente Figueiredo AM, por inacessibilidade de dados pluviométricos no referido município.

Os dados de variação do nível da água subterrânea foram obtidos a partir da rede integrada de monitoramento das águas subterrâneas (RIMAS) (http://rimasweb.cprm.gov.br/), conforme Tabela 1. A designação do tipo livre ou confinada dos aquíferos foi de acordo com as informações fornecidas pelo CPRM na descrição dos poços. A aquisição dos dados de nível 
e precipitação é de caráter diário. Nota-se que algumas séries apresentam descontinuidades de leitura que poderiam auxi- liar na contabilidade das recargas episódicas e anuais.

Tabela 1 - Poços selecionados para este estudo

\begin{tabular}{cccccccc} 
Poço & Lat & Long & Prof $(\mathrm{m})$ & $\begin{array}{c}\text { Prof inicial } \\
\text { dos filtros }(\mathrm{m})\end{array}$ & Aquífero & Tipo & Cidade \\
\hline P1 & 9656697 & 167156 & 24 & 6 & AC - Superior & livre & Manaus - AM \\
P2 & 9653077 & 169763 & 78 & 28 & AC - Superior & livre & Manaus - AM \\
P3 & 9660762 & 169524 & 80 & 28 & AC - Superior & livre & Manaus - AM \\
P4 & 9727565 & 754240 & 250 & 141 & AC - Inferior & livre & Santarém - PA \\
P5 & 9778250 & 824404 & 260 & 138 & AC - Inferior & confinado & Monte Alegre - PA \\
P6 & 015451 & 553101 & 115 & 82 & AC - Superior & confinado & Óbidos - PA \\
P7 & 9774734 & 830831 & 17.7 & 5.7 & Trombetas & livre & Presidente Figueiredo - AM \\
P8 & 9773316 & 202203 & 60 & 36 & Trombetas & livre & Presidente Figueiredo - AM \\
*Fonte: RIMAS & & & & & & &
\end{tabular}

Dados do satélite Grace (Gravity Recovery and Climate Experiment mission) para o período 2010 - 2017 foram oriundos do banco de dados do Koninklijk Nederlands Meteorologisch Instituut (KNMI) (https://climexp.knmi.nl). Estas informações foram utilizadas para comparar as variações do nível da água subterrânea dos poços ao déficit total no armazenamento de água para o período do El Niño 2014-2016.

\subsection{Método de estimativa de recarga}

Neste estudo, aplicou-se o método da Variação do Nível de Água (VNA), conforme definido por (HEALY E COOK, 2002), onde a elevação do nível de água em um aquífero livre é proveniente da adição de um volume de água ao mesmo. Para quantificar a recarga, é necessário multiplicar a variação do nível de água registrada em um intervalo de tempo pelo volume de poros que podem ser preenchidos pela água (Equação 01). Este volume, denominado rendimento especifico do aquífero (Sy), é a razão entre quantidade de água drenada pela gravidade de uma rocha ou solo, após ser saturada, e o seu volume total.

$R=S y \frac{\Delta h}{\Delta t}$

Onde Sy é o rendimento específico (unidade adimensional); $\Delta \mathrm{h}$ (mm) é a variação do nível de água, entendida como a diferença entre o ponto máximo na elevação do nível de água registrado no piezômetro e o ponto no mesmo tempo da curva extrapolada da última recessão; e $\Delta$ t é o tempo de medição decorrido (dias).

0 método é comumente aplicado a aquíferos superficiais que exibem acentuada flutuação de níveis. Nos aquíferos profundos estas variações tendem a se dispersar por longas distâncias, entretanto, não constitui um impedimento para aplicação do método em aquíferos confinados que exibam variações sazonais de recarga e descarga bem definidas. Normalmente, as taxas de recarga variam substancialmente devido a diferenças de altitude, arcabouço geológico, declividade da superfície, vegetação e outros fatores. Os poços devem ser localizados de forma que os níveis de água monitorados sejam representativos da bacia hidrográfica como um todo (HEALY e COOK, 2002).

O método também é amplamente difundido por gerar bons resultados, por ser de fácil aplicação e de uso irrestrito de qualquer tipo de poço (MALDANER, 2010). De acordo com Healy e
Cook (2002), a dificuldade de obter valores representativos de Sy é uma das limitações associadas ao método de VNA.

Em locais úmidos, como no caso da Amazônia, os intervalos entre as chuvas não devem exceder os tempos de resposta das águas subterrâneas, fato que pode tornar difícil a separação da ascensão freática. (NIMMO et al., 2014).

O delineamento de episódios específicos de recarga implica em julgamento caso a caso de forma gráfica, ou através de filtros automáticos (WU et al. 1996). Para esta pesquisa, optou-se em individualizar as recargas episódicas pelo método gráfico tendo como base episódios hidrológicos, onde pode-se estimar e corrigir a recessão não realizada (DELIN et al. 2007). 0 aumento efetivo devido a um episódio de recarga é considerado como a diferença entre a posição de pico do lençol freático e a recessão extrapolada no momento do pico. A extrapolação da recessão e a seleção dos momentos de início e término do evento requerem atenção manual para cada episódio (NIMMO et al., 2014).

Para o cálculo da recarga total anual, efetuou-se a extrapolação da curva de recessão, tomando-se o $\Delta h$, na intercessão entre valor máximo do nível d'água de cada ano e a extrapolação da curva de recessão.

Contabilizar todas as elevações associadas às precipitações pode gerar, segundo alguns autores, estimativas de recarga significativamente maiores do que outros métodos (WAHNFRIED e HIRATA, 2005). Para o método VNA, ressalta-se que as medições de nível da água devem ser frequentes e precisas (VARNI, et al., 2013).

Foram estimadas as recargas episódicas para cada um dos poços analisados, bem como suas recargas anuais. Os valores dos picos máximos e mínimos de nível da água também foram quantificados com relação ao total precipitado.

\subsection{Estimativa de rendimento específico do aquífero - Sy}

O principal desafio do método VNA envolve a correta estimativa do rendimento específico (Sy) do aquífero na profundidade da zona de flutuação do nível de água freático. Para estimar Sy aplicou-se o procedimento gráfico de Varni et al. (2013), o qual baseia-se no gráfico dos valores de precipitação versus os valores máximos de cada evento de subida de nível da água (NA). 0 inverso da inclinação de uma linha dese- 
nhada através da origem e acima de todos os pontos medidos dá o valor máximo do rendimento específico (VARNI et al., 2013). Este método de estimativa de Sy foi escolhido dada sua simplicidade e possibilidade de uso dos dados oriundos dos poços. Este método também permitiu comparação entre rendimentos específicos médios obtidos a partir de um furo de sondagem na FAC e as médias das macro porosidades até 1,20 m em diferentes posições de uma topo-sequência (baixio, vertente e platô), além de permitir a verificação dos respectivos erros de Sy por cada método.

\subsection{Correlação de Pearson}

A relação entre a precipitação e recarga foi avaliada em etapas de tempo anuais através do uso de gráfico de regressão e aplicação do coeficiente de correlação de Pearson segundo a seguinte fórmula:

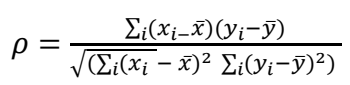

A análise de correlação indica a relação entre duas variáveis lineares e os valores sempre serão entre +1 e -1. 0 sinal indica a direção e o tamanho da variável indica a força da correlação.

\section{RESULTADOS E DISCUSSÃO}

\subsection{Variação do Nível da Água}

A Figura 2 mostra a variação do nível da água em função da precipitação diária acumulada. Os poços $\mathrm{P} 1$ a P6 representam a unidade aquífera AAC enquanto os poços $\mathrm{P} 7$ e P8, a unidade aquífera AT. Os poços P1 a P3 e P6 são considerados representativos da porção superior de AAC (até $130 \mathrm{~m}$ ), sendo P1 a P3 livres e P6 confinado. Os poços P4 e P5 encontram-se na porção inferior de AAC (>130 m), com filtros posicionados a partir de 142 m e 138 metros, e são considerados livre e confinado respectivamente. Os poços $\mathrm{P} 7$ e $\mathrm{P} 8$ representam a unidade aquífera AT em sua porção freática. Estes poços foram selecionados por possuírem séries históricas de monitoramento de nível da água (NA) mais extensas e responderem bem as variações sazonais da precipitação, conforme observado na Figura 2.

Figura 2 - Nível da água dos poços x precipitação para os aquíferos Alter do Chão Superior e Inferior e Trombetas

(a) P1 - AAC superior, filtros a partir de $6 \mathrm{~m}$ e intervalo de NA de $11 / 2010$ a $12 / 2018$.

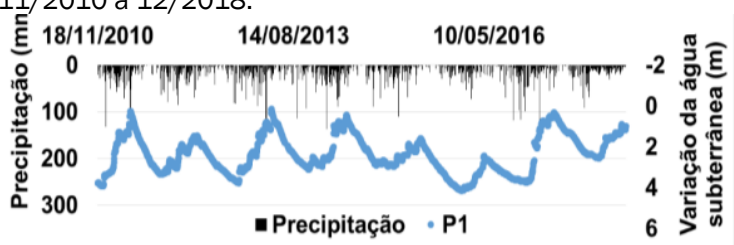

(c) P3 - AAC superior, filtros a partir de $31 \mathrm{~m}$ e intervalo de NA de $07 / 2012$ a 07/2018.

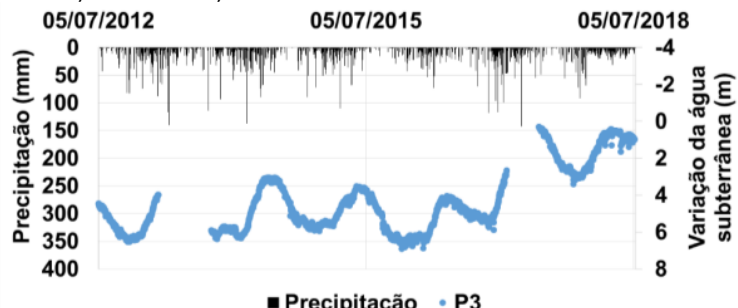

(e) P5 - AAC Inferior, filtros a partir de $138 \mathrm{~m}$ e intervalo de NA de 06/2013 a 07/2016.

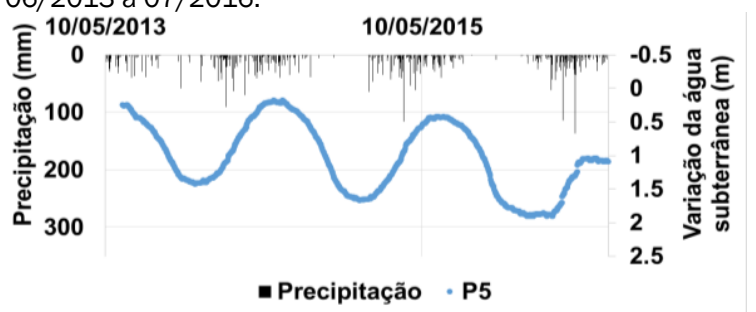

(g) P7 - AT, filtros a partir de $5 \mathrm{~m}$ e intervalo de NA de 03/2013 a $07 / 2018$.

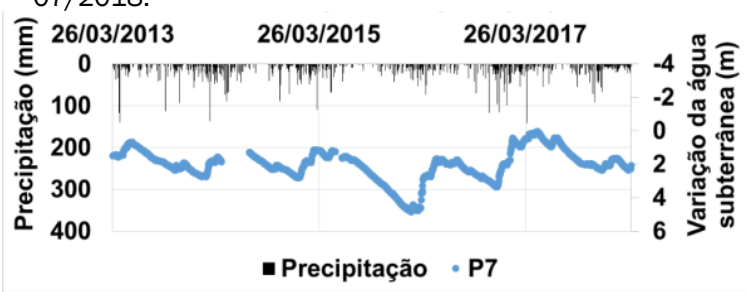

(b) P2 - AAC superior, filtros a partir de $28 \mathrm{~m}$ e intervalo de NA de 07/2012 a 07/2018.

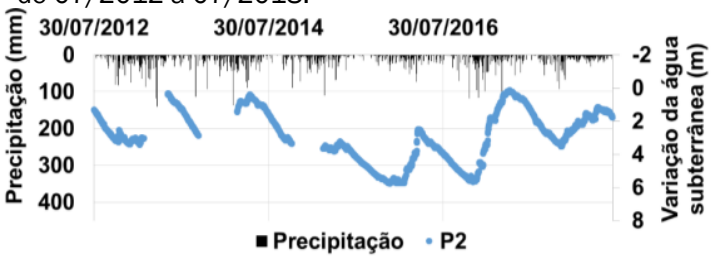

(d) P4 - AAC Inferior, filtros a partir de $142 \mathrm{~m}$ e intervalo de NA de 03/2013 a 04/2018.

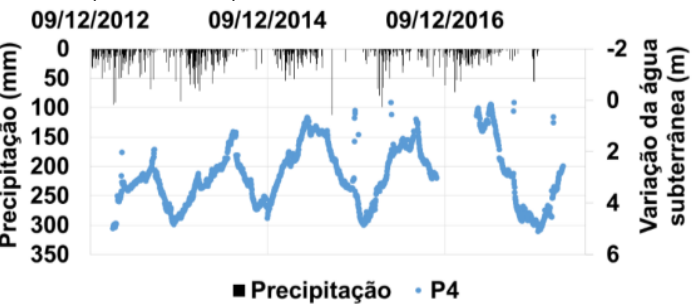

(f) P6 - AAC superior, filtros a partir de $82 \mathrm{~m}$ e intervalo de NA de 06/2014 a 12/2018.

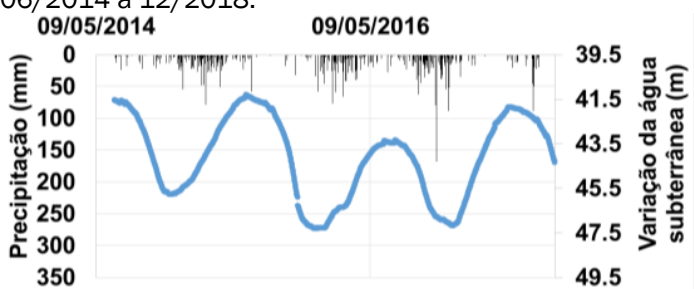

- Precipitação · P6

(h) P8 - AT, filtros a partir de $36 \mathrm{~m}$ e intervalo de NA de 03/2013 a $07 / 2018$.

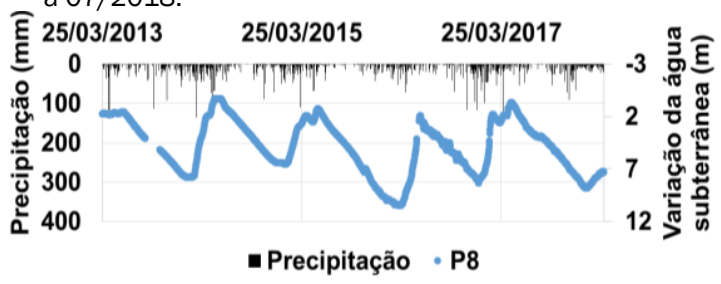




\subsection{Estimativa de Sy}

Com relação à determinação do Sy, aplicando-se o procedimento gráfico de Varni et al. (2013), obteve-se os valores de 0,27 e 0,08 respectivamente para as unidades AAC e AT (Figura 3). Se ressalta que a unidade AAC é constituída por arenitos médios a grossos, siltitos, argilitos e conglomerados enquanto que a AT, por arenitos finos a médios, siltitos, diamictitos e folhelhos. Tal diferença litológica reflete na vazão específica, uma vez que a porosidade é um termo determinante para Sy. Para os cálculos de recarga na AAC, entretanto, optou-se por utilizar o Sy de 0,17 (TOMASELLA et al., 2008), uma vez que este valor produziu o menor erro relacionado ao cálculo de recarga episódica, quando comparado aos erros gera- dos com o uso de Sy de 0,20 e 0,27 (Tabela 2). 0 método gráfico foi aquele que gerou o erro percentual $(37,04 \%)$ mais pronunciado e tendeu a superestimar Sy para AAC. Tomasella et al. (2008) obtiveram resultados de Sy a partir de análise de porosidade em furos de sondagem da PETROBRAS (Petróleo do Brasil S\&A), enquanto Brito et al. (2019), basearam-se nas médias das macro porosidades na porção superior de AAC, até $1,2 \mathrm{~m}$. Para este trabalho, todos os máximos episódicos de recarga foram contabilizados. É preciso lembrar que esta análise foi realizada para poços de profundidades e relações intraformação diferentes, o que também contribui para o aumento do erro. Como para a unidade AT não se conhecem valores de Sy para possível comparação, aplicou-se o valor de 0,08 obtido neste estudo.

Tabela 2 - Rendimento específico (Sy) e respectivos erros percentuais estimados

\begin{tabular}{c|ccc}
\hline Formação & Sy & Referência & Erro Porcentual \\
\hline Alter do Chão & 0,17 & Tomasella et al., 2008 & 17,66 \\
Alter do Chão & 0,20 & Brito et al., 2019 & 22,73 \\
Alter do Chão & 0,27 & Presente trabalho & 37,04 \\
\hline
\end{tabular}

Figura 3 - Valores de Sy para o AAC e o AT

(a)Sy Aquífero Alter do Chão

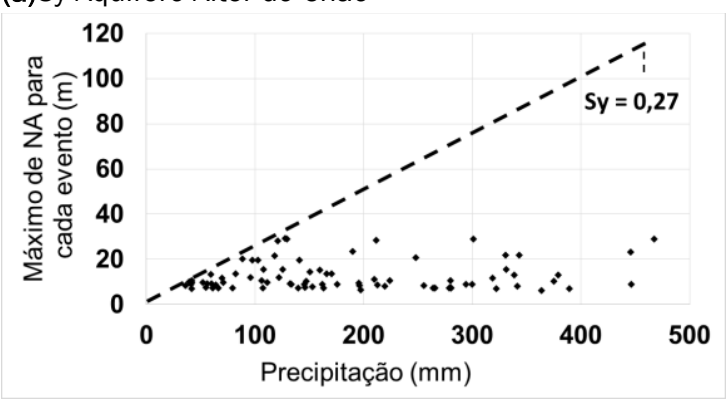

\subsection{Recarga Anual}

A Tabela 4 contém as estimativas anuais de recarga para os valores de Sy de 0,17 (ou 17\%) e 0,08 (ou 8\%) mencionados na seção anterior para as unidades AAC e AT, respectivamente. No P1 (AAC) ficou demonstrado, que para o período 2014-2015 e 2015-2016, houve uma diminuição gradual no total recarregado para o aquífero: respectivamente de $28 \%$ para $21 \%$. A maior taxa de recarga foi a do P2 (AAC), em torno de $49 \%$ ( $867 \mathrm{~mm}$ ) para uma precipitação anual de $1774 \mathrm{~mm}$ no período 2016-2017. Para o P3 (AAC) foi obtida apenas uma taxa de recarga (2015-2016), que corresponde a 393mm (26\%) do total anual precipitado de $1508 \mathrm{~mm}$.

Para o P4 (AAC), a taxa de recarga foi de 508mm (36\%) de um total precipitado de $1415 \mathrm{~mm}$ em $2014-2015$. No período seguinte, 2015-2016, a uma precipitação de $1111 \mathrm{~mm}$, a taxa de recarga foi de $415 \mathrm{~mm}$ (47\%). Em P4 houve um acréscimo de $1 \%$ da taxa de recarga entre os períodos, embora o período
(b)Sy Aquífero Trombetas

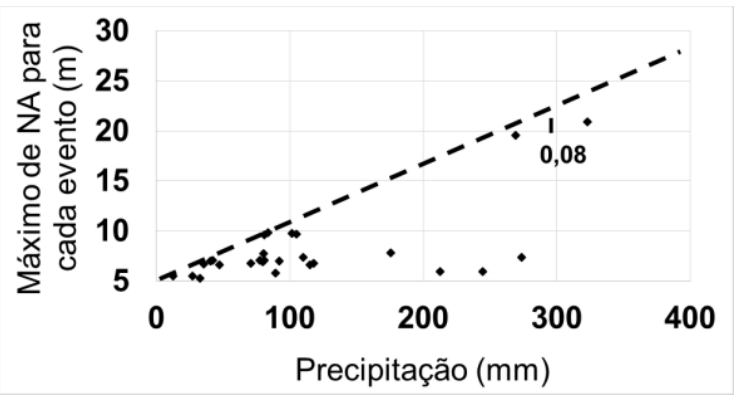

2015-2016 tenha precipitado $304 \mathrm{~mm}$ a menos em comparação a 2014-2015.

Em P5 (AAC), a recarga anual de 31\% foi a menor (529mm) a um total precipitado de $1695 \mathrm{~mm}$ no período 2014-2015 e representou um decréscimo de $2 \%$ do total recarregado quando comparado ao intervalo anterior, 2013-2014, cujo total precipitado foi de $1552 \mathrm{~mm}$ a uma taxa de recarga de $505 \mathrm{~mm}$ (33\%). Para o P6 (AAC), a menor taxa de recarga $(647 \mathrm{~mm})$ ocorreu no período 2015 - 2016, a um total de $1604 \mathrm{~mm}$ precipitados, correspondendo a $40 \%$ do total precipitado.

No P7 (AT) a menor taxa de recarga ocorreu em 2014-2015, cerca de $11 \%(187 \mathrm{~mm})$ do total precipitado $(1716 \mathrm{~mm})$. O P8 seguiu a tendência do P7 e gerou para 2014-2015, uma recarga de $394 \mathrm{~mm}$ (23\%) de $1715 \mathrm{~mm}$ precipitados. Já para o ano seguinte (2015-2016), nota-se um aumento para $36 \%$ (494mm) do total precipitado (1748mm). 
Tabela 3 - Precipitação anual - P (mm), Recargas - R (mm), variação do nível anual (m), nível máximo anual (usado para calcular a recarga) (m), declínio do nível anual $(\mathrm{m})$ e Sy $=0,17$ e Sy $=0,08$.

\begin{tabular}{|c|c|c|c|c|c|c|c|c|c|}
\hline Poços & Ano & Aquífero & $P(\mathrm{~mm})$ & $\mathrm{R}(\mathrm{mm})$ & $\Delta \mathrm{h}(\mathrm{m})$ & $\mathrm{hmin}(\mathrm{m})$ & $\operatorname{hmax}(\mathrm{m})$ & Sy & $\mathrm{R}$ \\
\hline P1 & 2011-2012 & AC - Superior & 2260 & 717 & 4,22 & 11,70 & 7,48 & 0,17 & $32 \%$ \\
\hline P1 & $2012-2013$ & AC - Superior & 1722 & 736 & 4,33 & 10,50 & 6,17 & 0,17 & $43 \%$ \\
\hline P1 & $2013-2014$ & AC - Superior & 2036 & 687 & 4,04 & 10,80 & 6,76 & 0,17 & $34 \%$ \\
\hline P1 & 2014-2015 & AC - Superior & 1716 & 483 & 2,81 & 10,50 & 7,76 & 0,17 & $28 \%$ \\
\hline P1 & $2015-2016$ & AC - Superior & 1379 & 294 & 1,73 & 10,20 & 8,47 & 0,17 & $21 \%$ \\
\hline P1 & $2016-2017$ & AAC - Superior & 1749 & 704 & 4,14 & 10,50 & 6,36 & 0,17 & $40 \%$ \\
\hline P1 & $2017-2018$ & AC - Superior & 2056 & 598 & 3,52 & 10,50 & 6,98 & 0,17 & $29 \%$ \\
\hline P2 & $2015-2016$ & AC - Superior & 1271 & 537 & 3,16 & 15,50 & 12,34 & 0,17 & $42 \%$ \\
\hline P2 & $2016-2017$ & AC - Superior & 1774 & 867 & 5,1 & 15,70 & 10,06 & 0,17 & $49 \%$ \\
\hline P3 & $2015-2016$ & AC - Superior & 1508 & 393 & 2,31 & 29,05 & 26,74 & 0,17 & $26 \%$ \\
\hline P4 & 2014-2015 & AC - Inferior & 1415 & 508 & 2,99 & 22,21 & 19,22 & 0,17 & $36 \%$ \\
\hline P4 & $2015-2016$ & AC - Inferior & 1111 & 415 & 2,44 & 23,25 & 20,81 & 0,17 & $37 \%$ \\
\hline P5 & $2013-2014$ & AC - Inferior & 1552 & 505 & 3,11 & 12,00 & 8,89 & 0,17 & $33 \%$ \\
\hline P5 & 2014-2015 & AC - Inferior & 1695 & 529 & 2,97 & 12,20 & 9,23 & 0,17 & $31 \%$ \\
\hline P6 & 2014-2015 & AC - Superior & 1508 & 680 & 4,00 & 45,50 & 41,5 & 0,17 & $45 \%$ \\
\hline P6 & $2015-2016$ & AC - Superior & 1604 & 647 & 3,00 & 47,28 & 43,05 & 0,17 & $40 \%$ \\
\hline P7 & 2014-2015 & Trombetas & 1716 & 187 & 2,34 & 8,50 & 6,16 & 0,08 & $11 \%$ \\
\hline P7 & 2015-2016 & Trombetas & 1379 & 242 & 3,03 & 9,77 & 6,14 & 0,08 & $18 \%$ \\
\hline P7 & 2016-2017 & Trombetas & 1749 & 333 & 4,16 & 9,60 & 5,44 & 0,08 & $19 \%$ \\
\hline P8 & 2013-2014 & Trombetas & 2036 & 586 & 7,32 & 24,6 & 17,29 & 0,08 & $29 \%$ \\
\hline P8 & 2014-2015 & Trombetas & 1715 & 394 & 4,92 & 23,41 & 18,49 & 0,08 & $23 \%$ \\
\hline P8 & $2015-2016$ & Trombetas & 1379 & 494 & 6,17 & 27,3 & 21,13 & 0,08 & $36 \%$ \\
\hline P8 & $2016-2017$ & Trombetas & 1748 & 601 & 7,51 & 25,23 & 17,72 & 0,08 & $34 \%$ \\
\hline
\end{tabular}

A média das precipitações e recargas para o AAC foi de, respectivamente, $1647 \mathrm{~mm}$ e $572 \mathrm{~mm}$, ou seja, em torno de $35 \%$ do total precipitado. $0 \Delta \mathrm{h}$ médio anual no $\mathrm{AAC}$ é de $3,5 \mathrm{~m}$. 0 índice de correlação de Pearson foi 0,8 , indicando uma forte correlação entre as precipitações e as recargas anuais obtidas.

No AT a média das precipitações foi de $1954 \mathrm{~mm}$ gerando recargas de $473 \mathrm{~mm}$, correspondendo percentualmente a $24 \%$ do total precipitado. $0 \Delta \mathrm{h}$ médio anual foi de $5.9 \mathrm{~m}$. Para o AT o índice de correlação de Pearson foi 0,4, indicando uma fraca correlação.

Brito (2014), usando o método VNA para o AAC em região de floresta, na Reserva Florestal Adolpho Ducke, Manaus-AM, estimou um valor de recarga em torno de $40 \%$ para o ano de 2013 e 32\% para o período 2014-2015, (Tabela 4). A adoção de valores diferentes de Sy pode ter conduzido a uma superestimação dos valores de recarga, fato que demonstra a sensibilidade do referido método para a variável Sy.

Tabela 4 - Comparação entre as taxas de recarga a partir de VNA para diferentes anos no AAC.

\begin{tabular}{|c|c|c|c|c|}
\hline Formação & Recarga \% & Método & Referência & Anos \\
\hline Alter do Chão & $40 \%$ & VNA & Brito (2014) & 2013 \\
\hline Alter do Chão & $32 \%$ & VNA & Brito et al., 2019 & 2014-2015 \\
\hline Alter do Chão & $35 \%$ & VNA & Presente trabalho & Média 2012 -2013 a 2015-2016 \\
\hline
\end{tabular}

No ano 2015-2016 há uma redução no total precipitado, fato que é acompanhado por uma tendência de redução da recarga iniciada em 2014-2015 (vide Tabela 3). Tal evento se deve ao ENOS em sua fase negativa (EI Niño - Oscilação Sul), que gera reduções nas precipitações da Amazônia (Ropelewski e Halpert 1987 e 1989), que para as estações aplicadas a este estudo resultaram em mais de 200 dias sem chuva no ano. Este fenômeno interanual iniciou em 2014, teve o ápice em dezembro de 2015, e perdurou até meados de 2016 (NOAA, 2019) (Figura 4a). Seus impactos sobre o armazenamento total de água ( $\mathrm{W}_{\mathrm{MI}}$ ) para a região geraram um rebaixamento significativo no total de massa de água $(-36 \mathrm{~cm})$ para o primeiro trimestre de 2016 (Figura 4b). Os poços captaram este déficit e os valores mínimos de nível da água ocorreram entre janeiro (P1, P2, P3, P7 e P8) a março (P4, P5 e P6) de 2016. 
Figura 4 - (a) El Niño e La Niña em seus máximos (https://climatedataguide.ucar.edu/climate-data/nino-sst-indices-nino-12-3-34-4-oni-and-tn). (b) Efeito de redução no armazenamento total de água consequentes da redução da precipitação ocasionada pelo El Niño 2015 para a região deste estudo obtidos através de dados de massa de água do GRACE Experiment

a) Sugere-se que o tempo de resposta entre o ápice do El Niño (Figura 5a) e o rebaixamento máximo dos aquíferos Alter do Chão e Trombetas seja de aproximadamente um mês meses. Os poços no AAC possuem resposta mais El Niño e La Niña moderados e fortes, com destaque para o El Niño de 2015, tido como muito forte (NOOA, 2019).

ONI: 1950-2018: Base: 1960-1989

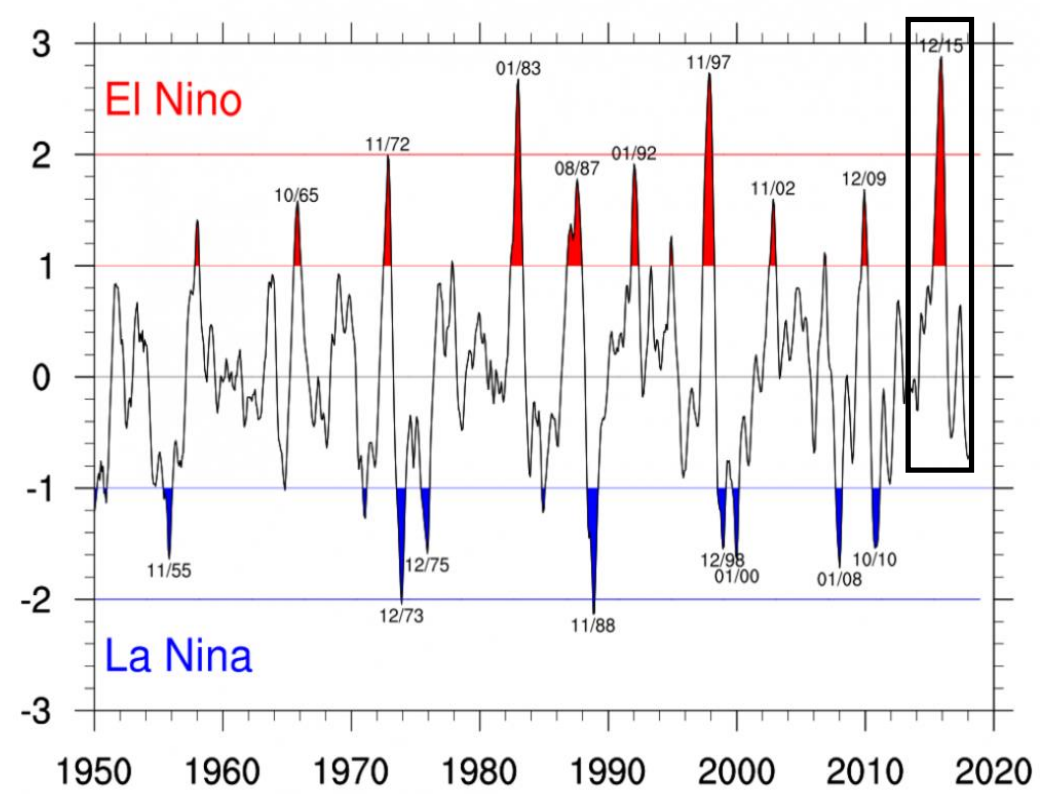

b) Efeitos do El Niño de 2015 no armazenamento total de água.

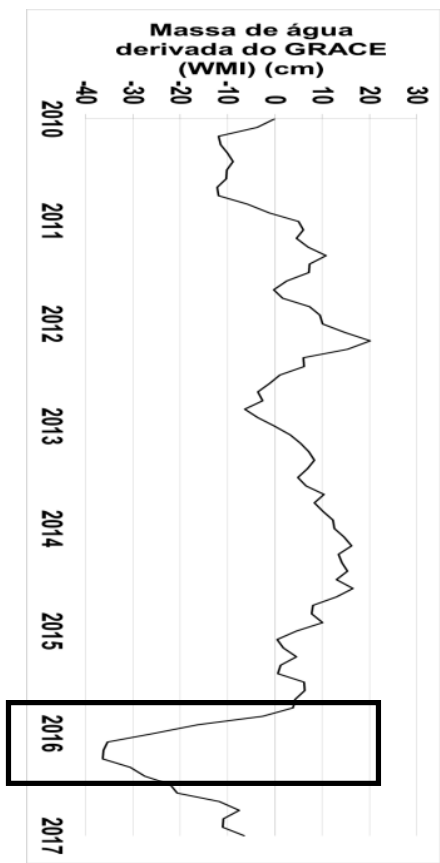

Estudos com base no El Ninõ 2005 indicaram que o evento também reduziu o total de água subterrânea armazenada no Aquífero Alter do Chão (FRAPPART et al., 2019) e que tal mecanismo é suportado pela persistência no déficit de chuvas no armazenamento subterrâneo (BIERKENS \& VAND DEN HURK, 2007), à semelhança do observado para o El Niño 2015 no presente trabalho.

\subsection{Recargas episódicas}

A Figura 5 mostra a relação entre as estimativas de recarga episódicas e as precipitações para o período compreendido entre 2011 e 2018. No P1 (Figura 5a), cuja série temporal apresenta poucas falhas, foi possível extrair cerca de 50 episódios de recarga (o máximo de episódios dentre os oito poços). Para este caso, portanto, a correlação de Pearson entre as recargas e o precipitado foi igual forte $(0,88)$. No P2 (Figura $5 b)$ e no P3 (Figura 6c) as correlações foram de 0,89 (forte) e 0,94 (muito forte).

A soma das seções filtrantes em cada um dos poços P1, P2 e P3 é de $18 \mathrm{~m}, 30 \mathrm{~m}$ e $32 \mathrm{~m}$, respectivamente. Embora as profundidades e a posição dos filtros sejam diferentes, há uma boa correlação entre as recargas estimadas e as precipitações para poços de até $80 \mathrm{~m}$ de profundidade. Uma sequência temporal maior auxiliará em futuras análises de correlação.

Os poços P4, P5 e P6 (Figuras 5d, 5e e 5f) são considerados profundos: $250 \mathrm{~m}, 260 \mathrm{~m}, 115 \mathrm{~m}$, com seções filtrantes a $141 \mathrm{~m}, 138 \mathrm{~m}$ e $82 \mathrm{~m}$ e com extensão de $40 \mathrm{~m}, 41 \mathrm{~m}$ e $33 \mathrm{~m}$; sob condição livre (P4) e confinada (P5 e P6) em função da presença de intercalações argilosas lenticulares (SOARES, WAHNFRIED e DINO, 2016).

Nestes poços a correlação entre recarga e precipitação foi de 0,69 (P4), 0,79 (P5) e 0,53 (P6), variando, portanto, de moderada a forte e de poucos episódios de recarga ( $\leq 14$ para cada poço).

Em P7 e P8 (figuras 5g e 5h) (AT), de profundidades 17,7 e $60 \mathrm{~m}$, as correlações foram muito fortes $(0,9$ e 0,92$)$. 0 poço P7 é o que possui a maior série temporal e o maior número de episódios de recargas (27 episódios). 
Figura 5 - Eventos de recarga distribuídos por poço e as respectivas correlações. a) Eventos de recarga para o período 2010-2018. b) Eventos de recarga no intervalo 2012-2018. c) Eventos de recarga entre o período 2013-2016. (d) Eventos de recarga entre 2013 a 2018. e) Recarga por eventos entre 2013 a 2016.f) Eventos de recarga entre 2014 a 2016 para a Formação Alter do Chão. g) Eventos de recarga entre 2014 e 2018 para o grupo Trombetas. h) Eventos de recarga entre 2013 a 2018 para o Grupo Trombetas

(a) Poço 1 - Formação Alter do Chão

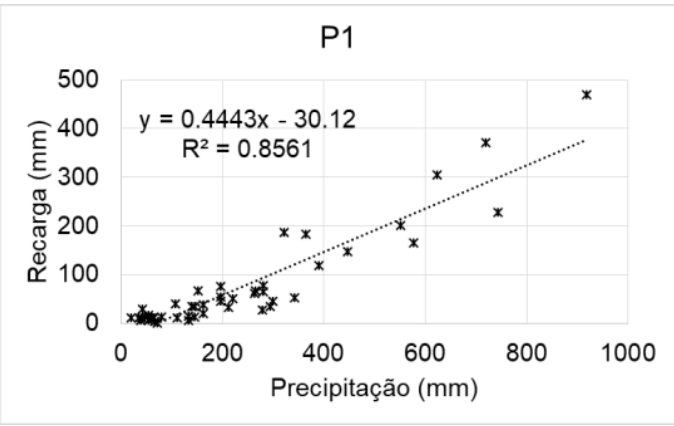

(c) Poço 3 - Formação Alter do Chão

P3

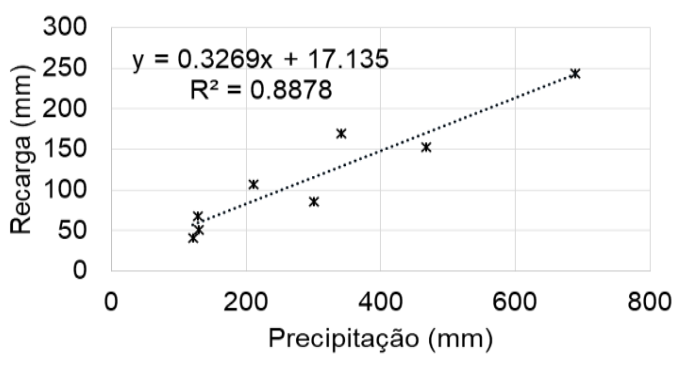

(e) Poço 5 - Formação Alter do Chão

P5

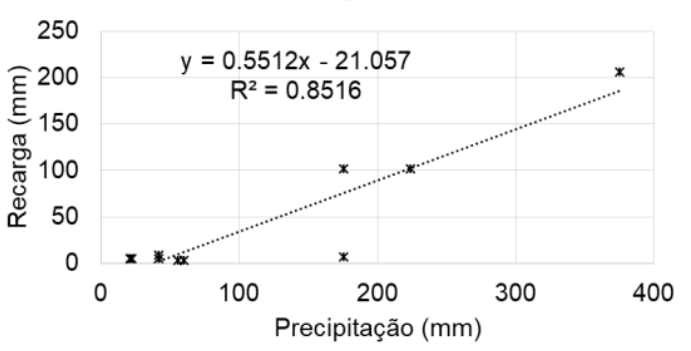

(g) Poço 7 - Grupo Trombetas

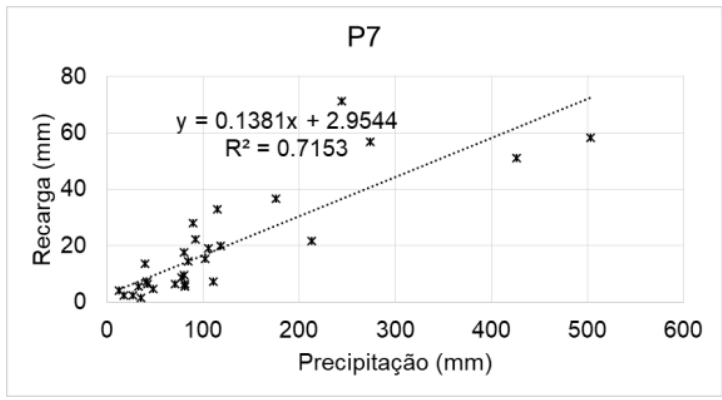

\section{CONCLUSÕES}

Para a unidade AAC a recarga média anual estimada foi de $35 \%$, correspondendo a $572 \mathrm{~mm}$ de $1634 \mathrm{~mm}$ médios precipitados e um $\Delta$ h médio anual de $3,5 \mathrm{~m}$. A correlação entre recargas e precipitações anuais foi forte $(0,8)$. Na unidade AT a recarga média anual foi de $24 \%$ ( $473 \mathrm{~mm}$ ) de um total médio de precipitação de $1954 \mathrm{~mm}$. Para esta unidade o $\Delta \mathrm{h}$ médio anual é de 5,9m. A correlação anual foi fraca $(0,4)$. Já a correlação entre precipitação e recargas episódicas para a unidade AAC nos poços P1, P2, P3 foi de 0,8, enquanto que nos poços (b) Poço 2 - Formação Alter do Chão

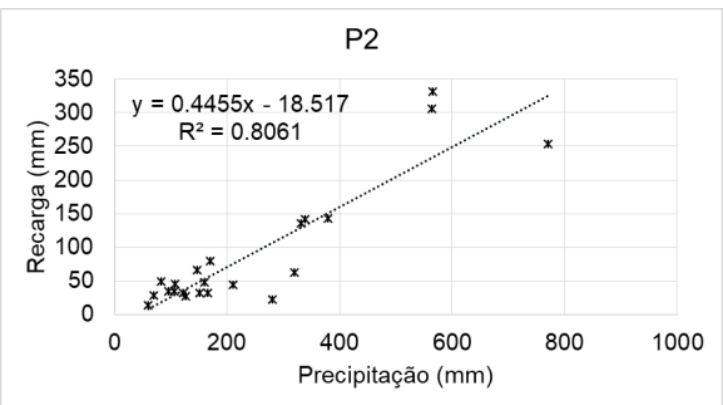

(d) Poço 4 - Formação Alter do Chão

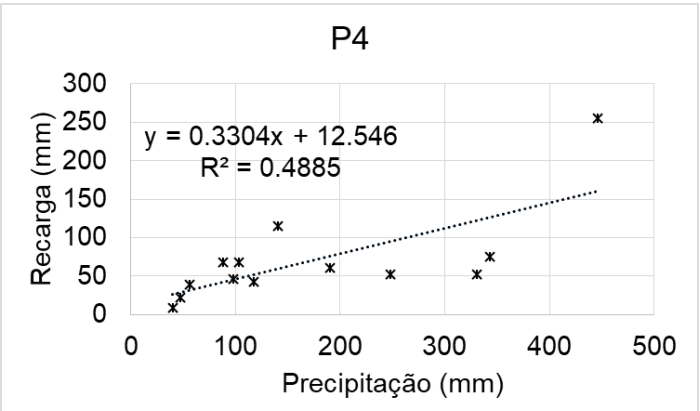

(f) Poço 6 - Formação Alter do Chão

P6

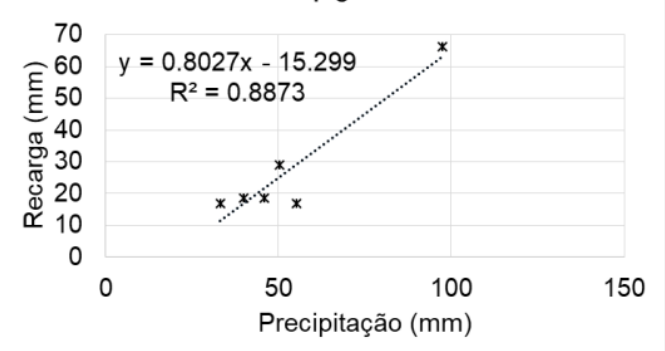

(h) Poço 8 - Grupo Trombetas

P8

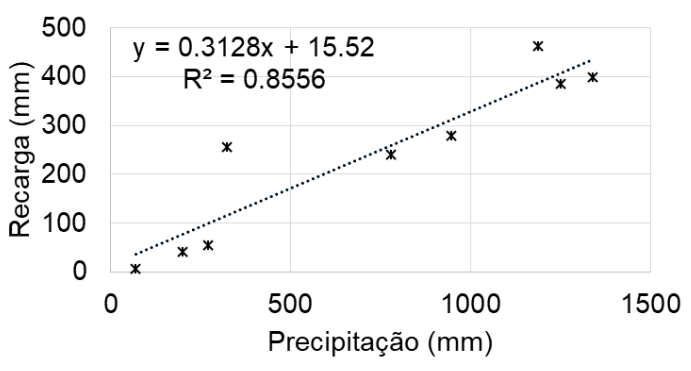

P4, P5 e P6, foi respectivamente de 0,6, 07 e 0,5. De forma geral, as correlações foram consideradas satisfatórias, inclusive em poços profundos (P4 e P5) e para os confinados P5 e P6. Na unidade AT as correlações foram muito fortes $(0,9)$. No poço $\mathrm{P} 1$, da unidade AAC, a série temporal mostrou-se como a mais extensa e coincidiu com os menores valores de precipitação (1379 mm) e recarga (294 mm, ou 21\% da precipitação). No AT, a menor a recarga foi de $11 \%(187 \mathrm{~mm})$ de 1716 precipitados para 2014-2015.

Para o período 2015-2016 no AAC, os poços P2, P3, P5 e P6, 
apresentaram recarga inferior à média dos períodos monitorados. A única exceção foi o poço P4, no qual houve indicação de aumento da recarga (1\%) no período 2015-2016. Com relação ao AT, os poços P7 e P8 apresentaram as menores recargas no período 2014-2015.

Sugere-se que esta diminuição na recarga esteja relacionada ao aumento de dias sem chuva (acima de 200 dias no montante anual) e a diminuição na concentração diária das chuvas. No caso da estação de Manaus, a média anual da precipitação entre 2010 a 2017 foi de $2443 \mathrm{~mm}$ sendo o ano de 2015 o de menor pluviosidade $(1828 \mathrm{~mm})$ e de maior quantidade de dias sem chuva para todas as estações, com uma redução de $615 \mathrm{~mm}$ comparado a média precipitada.

Isto significa que o efeito da diminuição da frequência de chuvas não gera uma diminuição linear na recarga, pois a recarga também é fortemente associada a fatores físicos intrínsecos ao arcabouço litológico de cada formação e das condições de uso do solo, podendo este efeito ser ainda maior.

Neste trabalho foi observada ainda a resposta da variação do nível da água subterrânea gerada pelo evento interanual El Niño 2015. A partir da persistência do déficit de precipitação, sucedeu-se uma afetação nas reservas subterrâneas. Sugerese que o tempo de resposta entre o ápice do El Niño de 2015 e o rebaixamento máximo do aquífero Alter do Chão seja de aproximadamente um mês. Para o AT foi de até três meses. 0 aumento nos dias sem chuva e a redução da precipitação anual contribui para reduzir o total de água armazenada nos aquíferos.

Somente a continuidade e expansão do programa de monitoramento de água subterrânea possibilitará análises com maior assertividade sobre a magnitude dos impactos em escala regional dos eventos interanuais nas águas subterrâneas na Amazônia.

\section{REFERÊNCIAS}

AGUIAR, C, J, B,; HORBE, M, A,; R FILHO, S, F,; LOPES, E, S,; MOURA, U, F,; ANDRADE, N, M,; DIÓGENES, H, S.Carta hidrogeológica da cidade de Manaus, Manaus, CPRM-AM, p, 1-4, (Relatório Interno). 2002.

ARAGÃO L., ANDERSON L.O., FONSECA M.G. 21st Century drought-related fires counteract the decline of Amazon deforestation carbon emissions. Nature Communications, 9, 536. 2018.

BAUM, C.A., MANCUSO, M. A., FRITZEN, R. R.Aplicação do método WTF no estudo da variabilidade da recarga em aquífero urbano. São Paulo, UNESP. Geociências, v. 37, n. 1, p. 85 98. 2018.

BIERKENS, M. F. P., \& VAN DEN HURK B. J. J. M. Groundwater convergence as a possible mechanism for multi-year persistence in rainfall..Geophys. Res. Lett. 34, L02402. 2007.

BLACK, W. C. Análise Multivariada de dados. Porto Alegre, Bookman. 2005.

BRITO, A. P.Elaboração de modelo numérico de fluxo de água subterrânea para uma microbacia de água clara na reserva florestal Adolpho Dcuke, Manaus - AM. Dissertação (Mestrado) - UFAM. 2014.

BRITO, A. P., WAHNFRIED, I., FERREIRA, S. J. F., BASTOS, J.H. (2019), Análise comparativa entre métodos de estimativa de recarga para uma microbacia na Reserva Florestal Adolpho Ducke, Manaus - AM. Geol, USP, Sér, Cient, São Paulo, no prelo.

CUNHA, P.R.C.; MELO, J.H.G.; SILVA, O.B.Bacia do Amazonas. Boletim de geociências. PETROBRAS. v. 15, p. 227-251.2007.

DE VRIES, J.J. AND SIMMERS, I. Groundwater Recharge: An Overview of Processes and Challenges. Hydrogeology Journal, 10, 5-17. 2002. http://dx.doi.org/10.1007/s10040-0010171-7

FAN;Y., LEE, H., MIGUEZ - MACHO, G. Global patterns of groundwater table depth. 2013. Disponivel em: http://science,sciencemag,org/content/339/6122/940.

FIGUEROA S., NOBRE C.A. Precipitation distribution over central and western tropical South America.Climanalise, n. 6, p. 36-40, 1990.

FRAPPART, F., PAPA, F., GUNTNER, A., TOMASELLA, J., PFEFFER, J., RAMILLIEN, G., EMILIOT, T., SCHIETTI, J., SEOANE, L., DA SILVA CARVALHO, J., MEDEIROS MOREIRA, D., BONNET, M.P., SEYLER, F.The spatio-temporal variability of groundwater storage in the Amazon River Basin. Advances in Water Resources, v. 124, p. 41-52. 2019. https://doi.org/10.1016/j.advwatres.2018.12.005

GLOOR M, BARICHIVICH J, ZIV G. Recent Amazon climate as background for possible ongoing and future changes of Amazon humid forests. Global Biogeochemical Cycles, 29, 1384 1399, 2015.

GREEN, T.R., TANIGUCHI, M., KOOI, H., GURDAK, J.J., ALLEN, D. M., HISCOCK, K. M. TREIDEL, H., AURELI, A. Beneath the surface of global change: Impacts of climate change on groundwater. J. Hydol.n. 405, p. 532 - 560. 2011. https://doi.org/10.1016/i.jhydrol.2011.05.002.

GURDAK, J.J., MCMAHON, P.B., BRUCE, B.W. Vulnerability of groundwater quality to human activity and climate change and variability, High Plains aquifer,USA. In: TREIDEL, H., MARTINBORDES, J.J., GURDAK, J.J. (EDS.), Climate change effects on groundwater resources: a global synthesis of findings and recommendations. Taylor \& Francis Publishing, Baca Raton, FL, pp. 145-167. 2012.

HEALY, R.W. \& COOK, P.G. Using ground-water levels to estimate recharge. Hydrogeology Journal, v. 10, p.10, p. 91 - 109, 2002.

KLOVE, B., ALA-AHO, P., BERTRAND, G., GURDAK, J.J., KUPFERSBERG, H., KVAERNER, J., MUOTKA, T., MYRKRA, H., PREDA, E., ROSSI, P., UVO, C. B., VELASCO, E., PULIDO-VELAZQUEZ, M. Climate change impacts on groundwater and dependent ecosystems. Journal of Hydrology, v. 518, p. 250266. 2014.

LERNER, D.N. Identifying and quantifying urban: a review. Hidrogeology Journal, v. 10, n. 1, p. 143 - 152. 2002.

MALDANER, C.Recarga de Aquífero em Área Urbana: Estudo de Caso de Urânia (SP), Dissertação (Mestrado) - Universidade de São Paulo, 2010. 
MARENGO J.A. e ESPINOZA J.C. Extreme seasonal droughts and floods in Amazonia: causes, trends and impacts. International Journal of Climatology, n. 36, p. 1033-1050, 2016.

MARENGO, J. A. Interdecadal variability and trends of rainfall across the Amazon basin.Theor. Appl. Climatol., n. 78, p. 7996. 2004. http://dx.doi.org/10.1007/s00704-004-0045-8.

MARENGO, J.A., BORMA, L.S., RODRIGUEZ, D.A., PINHO, P., SOARES, W.R., ALVES, L.M.Recent Extremes of Drought and Flooding in Amazonia: Vulnerabilities and Human Adaptation, American Journal of Climate Change, n. 02, p. 87-96, 2013.

NOAA. Disponível em: https://www.cpc.ncep.noaa.gov/products/precip/CWlink/MJO/enso.shtml. Acesso em: 03 nov. 2019.

PEREZ-VALDIVIA, C., SAUCHYN, D., VANSTONE, J. Groundwater levels and teleconnection patterns in the Canadian Prairies. WaterResour. Res. , n. 48, 2012. http://dx.doi.org/10.1029/2011wr010930.

PITA, R. C. S.; SILVA JÚNIOR, G.C.; ROSÁRIO, F.F.; SILVA, M. L. Sistema aquífero Alter do Chão a oeste da cidade de Manaus (AM): processos hidrogeoquímicos, origem da salinidade e relações com aquíferos adjacentes. Geol, USP, Sér, Cient,, São Paulo, v, 18, n, 1, p, 27-296. 2018.

ROPELEWSKI, C.F., HALPERT, M.S. Global and Regional scale precipitation patterns associated with the El Niño-Southern Oscillation. Monthly Weather Review, n. 115, p. 1606-1626, 1987.

ROPELEWSKI, C.F., HALPERT, M.S. Precipitation patterns associate al with the high index phase of Southern Oscillation. Journal of Climate, n. 2, p. 268-284, 1989.

SENEVIRATNE SI, NICHOLLS N, EASTERLING D. Changes in Climate Extremes and their Impacts on the Natural Physical Environment. In: Managing the Risks of Extreme Events and Disasters to Advance Climate Change Adaptation (eds Field CB, Barros V, Stocker TF, Qin D, Dokker DJ, Ebi KL, D MM, Mach KJ, Plattner GK, Allen SK, Tignor M, Midgley PM), pp, 109-230, Cambridge University Press, Cambridge, UK, and New York, NY, USA. 2012.

SOARES, E. A. A., WAHNFRIED, I., DINO, R. Estratigrafia de subsuperfície da sequência sedimentar cretáceaneógena das regiões de Manaus e Itacoatiara, Amazônia Central. Rev. Inst Geoc. USP, v.16, $\mathrm{n}$ 1, p. 23-41, 2016. DOI: 10.11606/issn.2316-9095.v16i1p23-41
STOLL, S., HENDRICKS FRANSSEN, H.J., BARTHEL, R., KINZEL$\mathrm{BACH}, \mathrm{W}$. What can we learn from long-term groundwater data to improve climate change impact studies? Hydrol. Earth Syst. Sci. n. 15, p. 3861-3875, 2011. 105194/hess-15-38612011.

TOMASELLA, J,; HODNETT, M,G,; CUARTAS, L, A,; NOBRE, A, D,; WATERLOO, M, J,; OLIVEIRA, S. The water balance of an Amazonian micro-catchment: the effect of interannual variability of rainfall on hydrological behaviour. 2008.

TREMBLAY, L., LAROCQUE, M., ANCTIL, F., RIVARD, C. Teleconnections and interannual variability in Canadian groundwater levels. J. Hydrol. n. 410, p. 178-188, 2011. http://dx.doi.org/10.1016/i.jhydrol.2011.09.013.

VAN CAMP, M. E WALRAEVENS, K. Impact of precipitation trends and the North Atlantic Oscillation on phreatic water levels in Low Belgium. GeologicaBelgica, n. 16/3, p. 157-163, 2013.

VARNI, M., COMAS, R., WEINZETTEL, P., DIETRICH, S. Application of the groundwater table fluctuation method to characterize groundwater recharge in the Pampa Plain, Argentina. Hydrological Sciences Journal, v. 58, n. 7, p. 1445-1455, 2013. https://doi.org/10.1080/02626667.2013.833663

VELASCO, E. M., GURDAK, J.J., DICKINSON, J.E., FERRÉ, T.P.A., CARONA, C. R. Interannual to multidecadal climate forcings on groundwaterresources of the U.S. West Coast. J. Hydrol. Regional studies, n. 11, p. 250-265, 2017. http://dx.doi.org/10.1016/i.ejrh.2015.11.018.

VENENCIO, M. DEL V., GARCÍA, N.O. Interannual variability and predictability of water table levels at Santa Fe Province (Argentina) within the climatic change context. J. Hydrol. n. 409,p. 62-70, 2011. http://dx.doi.org/10.1016/i.jhydrol.2011.07.039.

WADA, Y., VAN BEEK, L.P.H., VAN KEMPEN, C.M., RECKMAN, J.W.T.M., VASAK, S., BIERKENS, M.F.P. Global depletion of groundwater resources. Geophys. Res. Lett. n. 37, L20402. 2010.http://dx.doi.org/10.1029/2010GL044571.

WAHNFRIED, I., HIRATA, R. Comparação de métodos de estimativa de recarga de aquíferos em uma planície aluvionar na Bacia hidrográfica do Alto Tietê (São Paulo), Revista Brasileira de Recursos Hídricos, v, 10, n, 2, p, 15-25. 2005. 